Article title: Mother's dietary quality during pregnancy and offspring's dietary quality in adolescence:

follow-up from a nationwide birth cohort study of 19,582 mother-offspring pairs

Author names: Anne Ahrendt Bjerregaard, Thorhallur Ingi Halldorsson, Inge Tetens, Sjurdur Frodi Olsen

Affiliation and e-mail address of corresponding author

Center for Fetal Programming, Department of Epidemiology Research, Statens Serum Institut, Copenhagen,

Denmark, e-mail: anne@ssi.dk

\title{
S1 Figure.
}

Offspring median HEI score $\left(25^{\text {th }}-75^{\text {th }}\right.$ percentile $)$ as a function of maternal HEI score in quartiles. The HEI score could range from zero to 80 points.

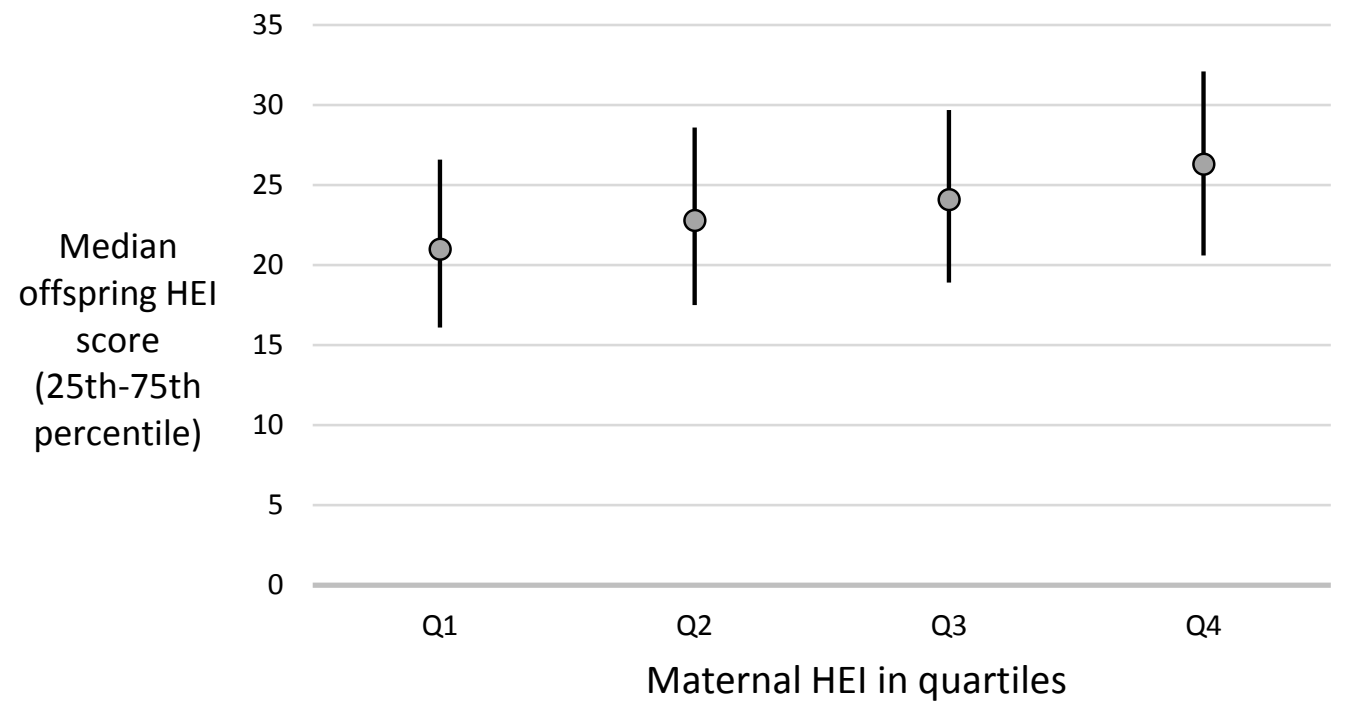

